\title{
A meta-analysis of channel switching approaches for reducing zapping delay in internet protocol television
}

\author{
Timothy T. Adeliyi, Ropo E. Ogunsakin, Marion O. Adebiyi, Oludayo O. Olugbara \\ ICT and Society Research Group \& Luban Workshop, Durban University of Technology, Durban, South Africa
}

\begin{tabular}{l} 
Article Info \\
\hline Article history: \\
Received Dec 28, 2020 \\
Revised Mar 21, 2021 \\
Accepted Apr 8, 2021 \\
\hline
\end{tabular}

\section{Keywords:}

Channel switching

Internet protocol

Meta-analysis

Television

Zapping delay

\begin{abstract}
Channel zapping delays are inconveniences that are often experienced by the subscribers of Internet protocol television (IPTV). It is a major bottleneck in the IPTV channels switching system that affect the quality of experience of users. Consequently, numerous channels switching approaches to minimize zapping delay in IPTV have been suggested. However, there is little knowledge reported in the literature on the determination of the strength of the evidence presented on the approaches of reducing zapping delay in IPTV, which is the prime purpose of this study. The extraction of the relevant articles was designed following the technique of preferred reporting items for systematic reviews and meta-analyses (PRISMA). All the included research articles were searched from the widely used databases of Google Scholar, and Web of Science. All statistical analyses were performed with the aid of the random-effects model implementation in Stata version 15 . The overall pooled estimated delay component was presented in forest plots. Overall, thirteen studies were included in the meta-analysis and the overall pooled estimate was $10 \%$ (95\% CI: 7\%, 30\%)). Experimental studies have shown that virtual elimination of IPTV zapping delay is possible for a relevant chunk of channel switching requests.
\end{abstract}

This is an open access article under the CC BY-SA license.

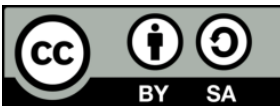

\section{Corresponding Author:}

Timothy T. Adeliyi,

ICT and Society Research Group \& Luban workshop,

Durban University of Technology,

Durban 4000, South Africa.

Email: timothya2@dut.ac.za

\section{INTRODUCTION}

The enhancement in the digital media industry has caused a paradigm shift for delivering innovative services to subscribers. This enhancement introduced a revolutionized era in media transmission by providing a bidirectional interactive feature in the digital media sphere. This new feature brought a tremendous increase to the demand for bandwidth in the modern age coupled with the roll-out of broadband convergence network and the delivery of high-definition video modes. The broadband convergence network transcends into three individual spheres: the media sphere (video), voice and data (Internet) which run in parallel and are amalgamated into a completely new bundle service.

A bidirectional interactive feature in televisions (TV) took an intrinsic benefit of the broadband convergence network by offering time-shifting, pay per view, video on demand and continuous interactivity to subscribers. Conversely, subscribers further encounter an inherent problem, zapping delay when changing channels. Because of the inherent issue, subscribers are forced to wait for several seconds before a TV channel becomes available. This is a crucial issue that deters terrestrial subscribers from converting and hence preventing the widespread adoption of internet protocol television (IPTV) services [1]-[3]. 
The Internet protocol television provides two major network infrastructures firstly, the managed IPTV network infrastructure provides a service model in which network service providers commit a qualityof-service network bandwidth for everything from the delivery of digital TV content to storage management, packet prioritization and bandwidth reservation methods in order to provide subscribers with a seamless quality of experience $(\mathrm{QoE})$. Furthermore, the reduction of bandwidth restrictions due to high demand that leads to packet delays and jitters confronted by peer to peer (P2P) and over-the-top (OTT) application services is reduced when a managed service infrastructure is implemented [4]-[5]. The IPTV managed service offers video content features that are provided by a service provider that operates an IPTV service or has arrangements with digital video content providers to deliver digital content via the set-top box (STB). As a result, providing a reserved and guaranteed bandwidth that enhances IPTV services provided to subscribers [6]-[7].

The unmanaged network infrastructure adopts the hypertext transfer protocol (HTTP) that gives subscribers to online television access. Online television is also known as Internet TV use the transmission control protocol (TCP) as reliable means to transmit OTT contents and to create a connection with the servers. The unmanaged network infrastructure offers best-effort open Internet access that gives no provisioning for quality of service because of the erratic availability of bandwidth at a given period. Various unmanaged network OTT video streaming applications allows the OTT content to be easily viewed through a web browser. However, anomalies encountered in an unmanaged network infrastructure reveal that without any preferential treatment in guaranteeing bandwidth from the network providers hampers the network performance metrics such as packet loss, delay and jitter.

\section{RELATED WORKS}

Over the years, several methods have been proposed in reducing the zapping delay for both managed and unmanaged network infrastructures in IPTV. Table 1 presents some proposed channel switching methods in the literature which includes methods that substitute multicast-based digital video packets with a unicast-based digital video packet [8]-[9]. Despite benefits multicast-based IPTV proffers such as saving network bandwidth, lowering network congestion and saving on server load, according to Škrbić et al. [10] a major limitation of the multicast-based service is that all deployed network devices must have Internet group management protocol (IGMP) capabilities has IGMP is used for managing multicast data. This limitation is further heightened by the high cost of implementation because of compatibility issues among different network device vendors and the complexity of troubleshooting multicast network services hence, decreasing quality of service. Contrary to the deployment of a multicast-based service system Alasaad et al. [11] proposes a "collaborative media streaming in community networks" approach to forecasting future media content demand that aims to leverage the resilience and excess accessible network micro-resources in a community network to create a virtual aggregate macro-resource. However, their approach does not elucidate the challenges faced when streaming live media content.

According to Lee et al. [12], their strategy focused on the subscriber level. They demonstrated a method for identifying hot channels, or channels that are likely to be viewed in the near future by an IPTV viewer. Their strategy dynamically preserves a list of hot TV channels and a list of cold TV channels at the subscriber level, the latter of which is unlikely to be watched anytime soon by the IPTV subscriber. However, the division of IPTV channels into hot and cold lists, on the other hand, is focused on channel popularity among IPTV subscribers as well as the subscribers' viewing history. During channel switching, the presented approach's hot list is checked first, in order to enable a preview mode hence, hot channels are obtained prior in low resolution.; these features are in place to significantly minimize zapping latency, even if network bandwidth is reduced.

Furthermore, Merani et al. [13] proposed a subscriber level approach in a peer-to-peer (P2P) network, that learns beforehand about the subscriber channel preference while monitoring their behavior. The video chunk scheduling algorithm of the overlay is changed to represent regular subscribers preferentially, providing them with a better viewing experience and quicker access to the selected channel without unnecessarily penalizing less habitual subscribers, in order to cluster them in groups that display different habits. However, subscribers that do not watch TV channels regularly are not catered for with this approach. Ghaderzadeh et al. [14] claimed that the approach presented by Merani et al. [13] causes network overload. As a result, they proposed ReDepoly, a distributed intelligence approach in a P2P network that replaces agents on the networks with bootstrapping peers to model user behaviors and share aggregated information between the agents to disseminate the learned models. Their approach helped reduce zapping according to presented results, hence increasing the quality of service.

Malkoş et al. [15] presented a channel-based peer selection approach where an IPTV subscriber in a peer-to-peer network constructs a peer-to-peer communication and uses a unicast stream to join a newly requested channel from a peered subscriber during zapping delay hence, avoiding a black screen during a 
channel request. Ghahfarokhi et al. [16] proposed an approach at the network level, their approach exploits the in-network caching feature of the information-centric networking to retrieve packet frames from the network. Hence, reducing synchronization and buffering delays without hampering on video quality received and maintaining bandwidth utilization.

Adeliyi and Olugbra [17] presented an approach that helps reduce zapping delay at the subscriber level. They devised a technique known as the two-list group program-driven algorithm, which reduces channel seek distance and time. Their method groups common channel program categories such as news, movies, sports, music, and documentaries to make it easier for channels with similar channel programs to be quickly identified among the many channels available on an internet protocol television. It was concluded that the simulated results showed an interesting result in reducing zapping delay by $58 \%$.

Chen and Chiu [18] had earlier presented a channel interleaved reordering based on user's interest (CIRI) to reduce the zapping time problem. They used channel reordering and preload policy to classify genre for channels to shorten seek distance and zapping time. Sarni et al. [19] further presented an approach at the network level called the channel switching scenario in Multicast IPTV Network. Their method switches between channels by sending a join message to the desired channel before leaving the one being watched. They tried to minimize channel zapping time in the network core without increasing multicast bandwidth usage. Using meta-analysis random-effects models, the overall objective of this study was to provide a review of channel switching approaches that would help to minimize zapping delay in IPTV.

Table 1. Channel switching methods

\begin{tabular}{|c|c|c|c|c|c|c|c|}
\hline Authors & Delay component & $\begin{array}{c}\text { IPTV } \\
\text { architecture }\end{array}$ & Video Coding & $\begin{array}{l}\text { Number of } \\
\text { subscribers }\end{array}$ & $\begin{array}{c}\text { Channel } \\
\text { switching } \\
\text { delay (ms) }\end{array}$ & $\begin{array}{c}\text { First } \\
\text { switching }\end{array}$ & $\begin{array}{l}\text { Stream with } \\
\text { min quality }\end{array}$ \\
\hline [17] & Subscriber & Managed & MPEG/H.264 & 1 & 25 & No & No \\
\hline [19] & Network & Managed & MPEG/H.264 & $32-512$ & $20-200$ & Yes & No \\
\hline [20] & Network & Managed & MPEG/H.264 & 20 & $56-90$ & Yes & No \\
\hline [2] & Network & Managed & MPEG/H.264 & 20 & 27 & No & No \\
\hline [23] & $\begin{array}{c}\text { Synchronization }+ \\
\text { buffering }\end{array}$ & Managed & MPEG/H.264 & - & - & Yes & Yes \\
\hline [24] & $\begin{array}{l}\text { Synchronization }+ \\
\text { buffering }\end{array}$ & Managed & MPEG/H.265 & 1 & $5-10$ & Yes & No \\
\hline [29] & All & Managed & MPEG/H.264 & 250000 & $1 e+7$ & No & No \\
\hline [30] & All & Managed & $\begin{array}{c}\text { SVC } \\
\text { (H.264/AVC) }\end{array}$ & 1712 & - & No & Yes \\
\hline$[31]$ & All & Managed & $\begin{array}{c}\text { SVC } \\
\text { (H.264/AVC) }\end{array}$ & 1 & 673200 & Yes & Yes \\
\hline$[32]$ & All & $\mathrm{P} 2 \mathrm{P}$ & MDC & $5-2000$ & $5 e+6-2 e+7$ & Yes & Yes \\
\hline [33] & All & WiMAX & MPEG/H.264 & $20-140$ & $1.3 e+6-6 e+6$ & Yes & No \\
\hline [3] & All & Unmanaged & MPEG/H.264 & 1 & 400000 & Yes & No \\
\hline
\end{tabular}

\section{MATERIALS AND METHODS}

\subsection{Literature strategy}

This study was carried out following the preferred reporting items for systematic reviews and metaanalysis (PRISMA) [34]-[36]. In this systematic review and meta-analysis, we searched the Web of Science and Google Scholar databases for the relevant published articles. These databases were searched for English papers published between 2004 and 2019 using the following combination of terms. Internet Protocol Television (IPTV) and text words in Web of Science: ('zapping delay' [IPTV Terms] OR ('zap time'[All Fields] AND 'IPTV'[All Fields]) OR 'channel switching'[All Fields] OR 'channel switching delay'[All Fields] OR 'zap time'[IPTV Terms]) AND ('channel switching [IPTV Terms]. Using Boolean operators, the search words were isolated or merged such as "OR" or "AND". EndNote X9 was used to import the studies found using the search strategies. As shown in Figure 1, a total of 342 published papers were found between 2004 and 2019. Reference lists of the relevant studies from the literature were checked manually for any citations missed when searching the electronic database. 


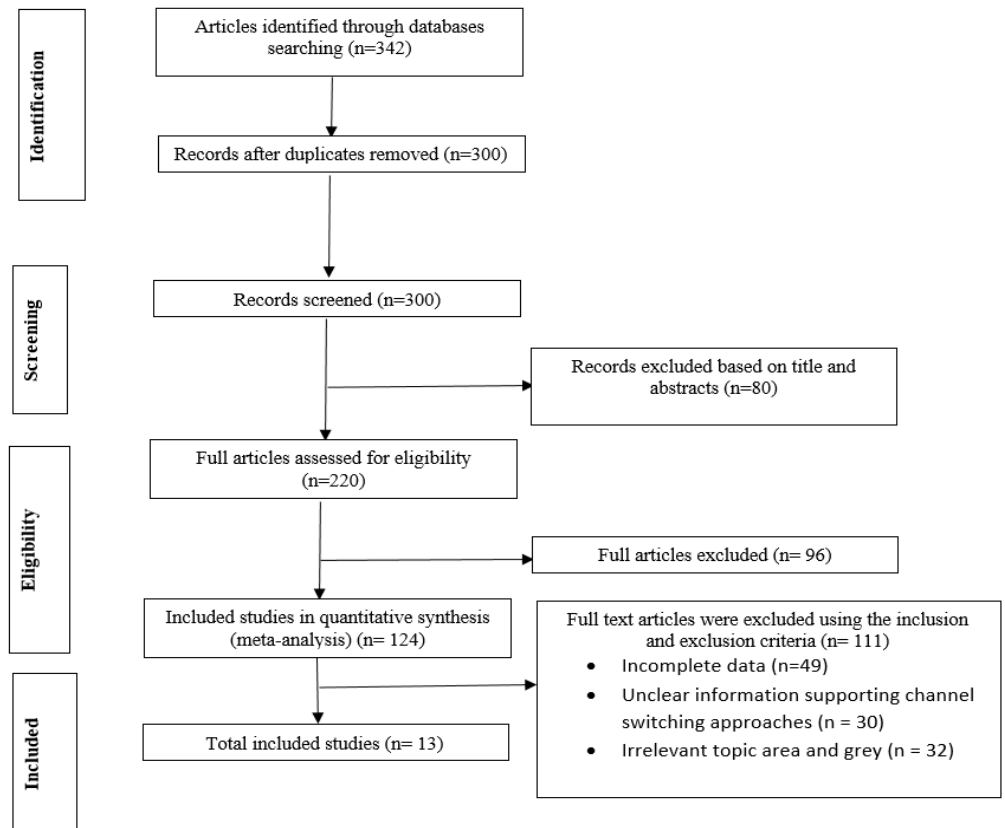

Figure 1. Flow diagram of the database searches (preferred reporting items for systematic reviews and meta-analysis)

\subsection{Inclusion and exclusion criteria}

The studies conducted to determine the delay components, the adopted IPTV architecture, video coding, video stream with minimal quality, multiple view state, next channel prediction, the total number of subscribers used in the analysis, and channel switching delay were the inclusion criteria for the papers. Articles with research designs such as analysis, letters to editors, editorials, commentaries, expert opinions, articles, book chapters, brief studies, and thesis published in dialects different from English before December 2003 were omitted. Also, articles that failed to report on the inclusion criteria were excluded.

\subsection{Quality assessment}

Information that met the inclusion criteria were extracted from the selected studies. The studies with channel switching delay in seconds (s) were manually converted to microseconds (ms) although some study simulations present their channel switching delay to $20 \mathrm{~ms}$. Also, some studies performed their simulation on a system without specifying the total number of subscribers, such studies were assumed to have used one subscriber in their simulation. The study with $100 \%$ information that fit into the inclusion criteria was appropriate for the systematic review and meta-analysis.

\subsection{Data synthesis and statistical analysis}

The information collected was inputted into an excel spreadsheet in preparation for the statistical analysis. Because heterogeneity is assumed, the random-effects model was used for the meta-analysis. The primary outcome was to estimate the pooled estimate of reducing zapping delay. To determine betweenstudy heterogeneity, the Cochrane Q statistic was used. $\mathrm{I}^{2}$ was used to assess heterogeneity among studies. The degree of heterogeneity between the included studies was assessed using the index of heterogeneity $\mathrm{I}^{2}$ values of $0 \%, 25 \%, 50 \%$, and $75 \%$, respectively, to reflect no, low, medium, and high heterogeneity. A DerSimonian and Laird random-effects model was used to create a pooled estimate for reducing zapping delay in IPTV. In addition, we performed a subgroup analysis centered on the research delay component to define the source of study heterogeneity. The existence of significant publication bias was evaluated through a funnel plot and Egger's regression test [37], [38]. For Egger's regression test, 0.05 was considered a meaningful value. Trim and fill study, suggested by Duval and Tweedie, enhances studies by making the distribution symmetrical. Small studies are omitted before the funnel plot is symmetrical, the true center of the funnel is estimated, and the excluded studies and their incomplete complements about the center are substituted. It estimates the number of missing studies, and an adjusted treatment effect is obtained by performing a meta-analysis, including the filled studies [37], [39]. We could not conduct meta-regression due to the insufficient information from the extracted articles that met the inclusion criteria. Stata-v12 was used for all statistical analysis. 


\section{RESULTS}

The findings in this study were obtained according to the items of the PRISMA, as shown in Figure 1. Following the initial search, 342 papers were found in two separate databases. Just 300 papers remained in the sample after EndNote X9 removed duplicates. After reading the abstracts, 80 papers were crossed out. Following the study of the papers, a total of 207 were removed for varying reasons. Finally, thirteen related papers published between 2004 and 2019 were chosen for final review based on the inclusion and exclusion criteria. However, only eight was selected for inclusion in the final meta-analysis since we took into account studies with multiple subscribers.

\subsection{Pooled estimate}

Studies included in this paper reported pooled estimates within the range of $1 \%$ to $87 \%$, with an overall (pooled) estimate of 19\% (95\% CI: 7-30\%, Figure 2). Since there was substantial heterogeneity across the studies $\left(\mathrm{I}^{2}=99.99 \%, \mathrm{p}=0.00\right)$, we used a random-effect model for the meta-analysis. Between-study variability was high (tau-squared $=0.03$ with heterogeneity chi-square $=56484.61$, a degree of freedom $=7$ ). Figure 2 presents the forest plot derived from the meta-analysis. The inference of $\mathrm{I}^{2}$ is that it represents the proportion of the total variance that can be attributed to the heterogeneity of actual study effects size. The interpretation of heterogeneity or homogeneity aids in important decisions, such as whether the collected studies are sufficiently similar to incorporate findings.

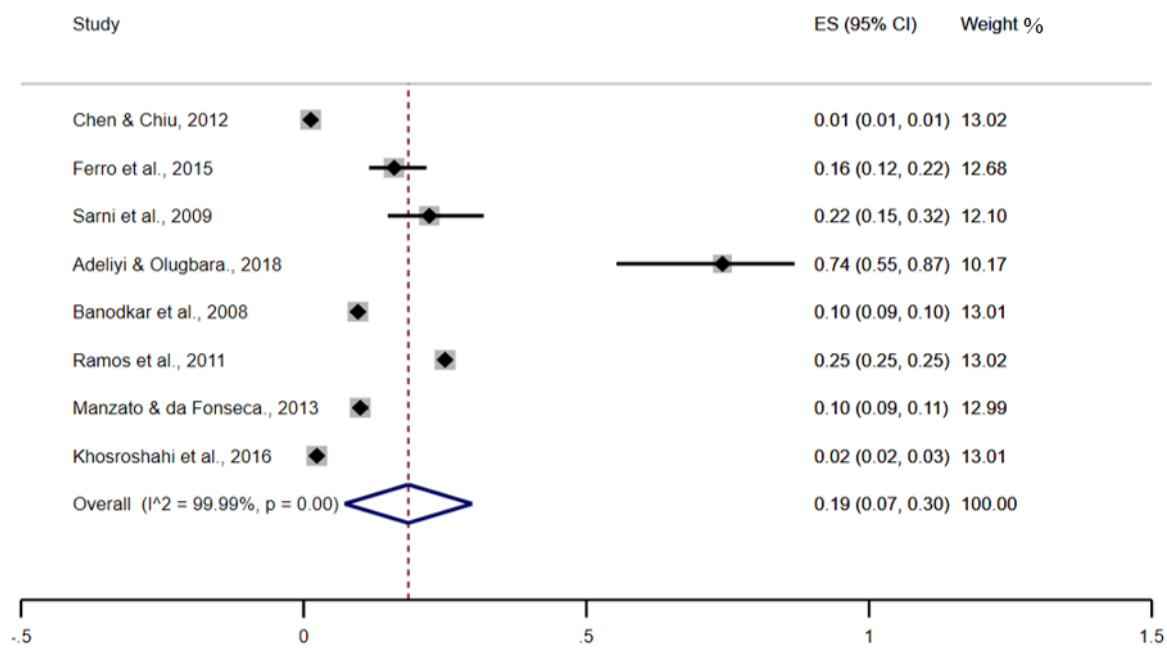

Figure 2. Graphical forest plot revealing the pooled estimate of techniques of reducing zapping delay using channel switching approaches

\subsection{Subgroup meta-analysis}

Furthermore, to minimize the random variations among the primary study's point estimates, subgroup analysis was performed to assess the potential heterogeneity between studies. Consequently, the result of subgroup analysis by delay component affirmed high variability in studies reporting a pooled estimate of reducing zapping delay (Higgins $\mathrm{I}^{2}$ statistic $=97.69 \%$ with heterogeneity chi-square $=129.65$, a degree of freedom $=3$ and $\mathrm{P}=0.001$ ) in the network. The sub-total random pooled estimate of reducing zapping delay was estimated at 26\% (95\% CI: 9-44\%) in the network and 12\% (95\% CI: 5-30\%) in studies that utilized all the delay component. The Forest plot is shown in Figure 3 and the overall statistics in Table 2. Therefore, the pooled effect estimate's validity for each subgroup is uncertain, as individual study results are inconsistent. The high heterogeneity could also be attributed to the number of subscribers' variation and how each study was conducted.

Table 2. Subgroup analysis for comparison of the pooled estimate of reducing zapping delay

\begin{tabular}{lcccccc}
\hline Delay component & No of studies & $\begin{array}{c}95 \% \mathrm{CI} \\
\text { estimate }\end{array}$ & $\mathrm{I}^{2}(\%)$ & $\mathrm{Q}$ & \multicolumn{2}{c}{ Heterogeneity test } \\
Df & p-value \\
\hline $\begin{array}{l}\text { Network } \\
\begin{array}{l}\text { Synchronization }+ \\
\text { buffering }\end{array}\end{array}$ & 4 & $26(9-44)$ & 97.69 & 129.65 & 3 & $<.0001$ \\
All & 1 & $10(9-10)$ & 0.00 & 0.00 & 0 & - \\
\hline
\end{tabular}




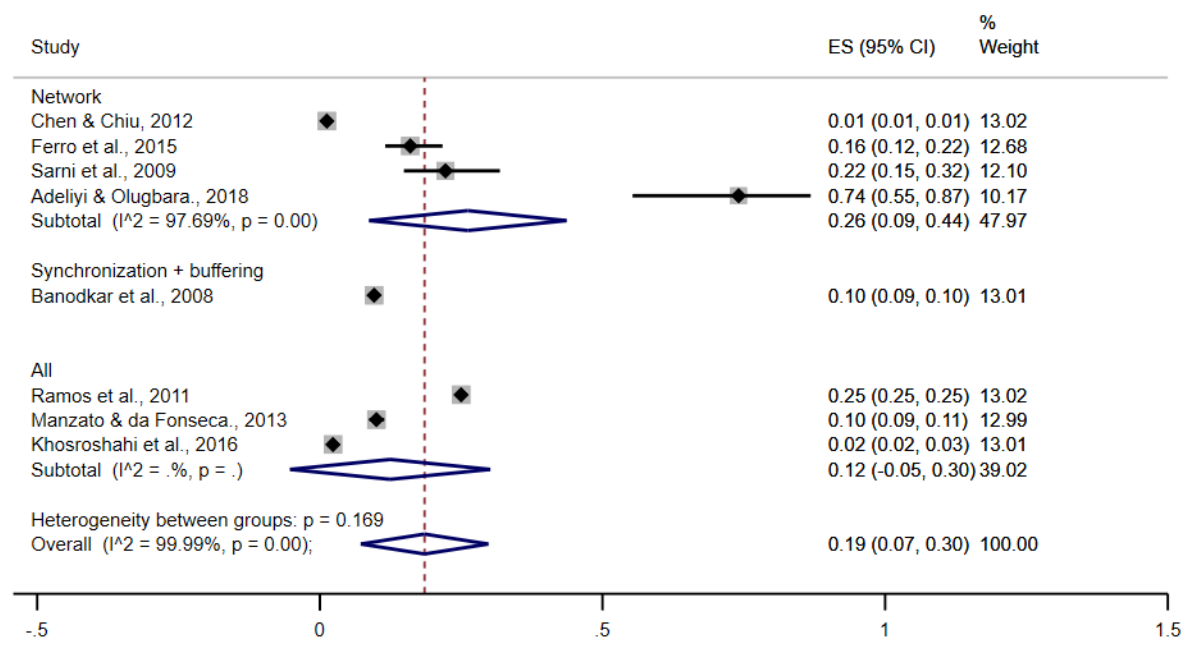

Figure 3. Forest plot on the pooled estimate of reducing zapping delay by a subgroup analysis in delay component

\subsection{Publication bias}

In systematic reviews and meta-analysis, publication bias is unavoidable [40]-[42]. It is also one of the key threats to the cogency of meta-analysis. It occurs when the outcome of a research study influences whether to publish or not. It may contribute to the development and testing of theories based on erroneous impressions gleaned from the scientific literature. Thus, publication bias's impact should be assessed to conclude the generalizability and restrictions of the collective findings. A publication bias review aims to determine the extent to which publication bias influences the summary outcome, and thus to assess the validity of the core findings. One standard method, whether there is any publication bias, uses funnel plots to identify such biasedness graphically. Hence, in this study, publication bias was examined using funnel plots and Egger tests. A funnel plot is a scatterplot in which the study's standard errors or sample sizes are plotted against the study's effect sizes. The visual inspection of the resulting funnel plot revealed an asymmetrical distribution of the study findings as shown in Figure 4. Each funnel plot represents a different sample, and an asymmetrical distribution indicates publication bias. The standard error of the estimation is represented on the $y$-axis. The logit transformed estimates are shown on the $x$-axis. First, each study's effect size was plotted against the standard error, and visual inspection of the funnel plot indicates some asymmetry. Asymmetry was visible in the funnel plot, indicating the existence of publication bias. The overall estimated effect size and its $95 \%$ confidence interval are shown by the vertical and diagonal dashed lines. The prevalence of publication bias was shown by the findings of the Begg's test $(\mathrm{z}=0.78, \mathrm{p}<0.05)$ and the Egger's test $(\mathrm{t}=5.89$, $\mathrm{p}$ value $=0.004)$. In addition, the funnel plot revealed an asymmetric distribution as shown in Figure 4 .

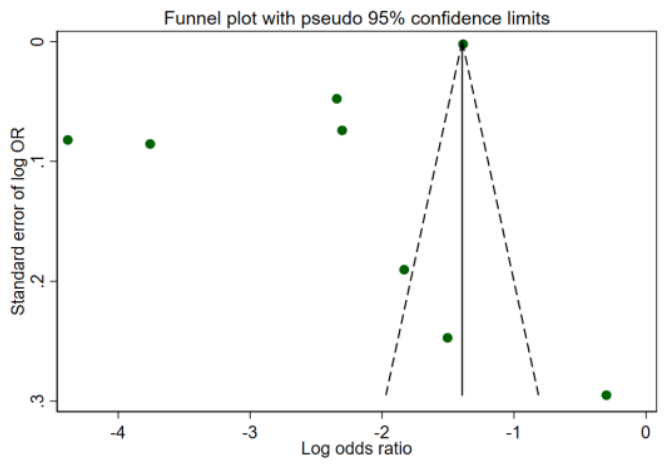

Figure 4. Funnel plot of publication bias

\section{DISCUSSION}

This section will discuss the analysis of the results of the recent works in reducing zapping delay in internet protocol television. This meta-analysis is based on four major channel switching approaches in the literature that has been proposed to reduce zapping delay. As shown in Table 1 delay component approaches

A meta-analysis of channel switching approaches for reducing zapping delay in ... (Timothy T. Adeliyi) 
such as subscriber, network, synchronization and buffering, and an approach that combines all previously mentioned approaches (All) in reducing zapping delay was considered from 2004 to 2019. For an accurate result, our analysis only considered eight kinds of literature out of the thirteen kinds of literature that meet the inclusion criteria. The selected eight kinds of literature simulated there a method for more than one user hence giving more credibility to the result reported

When comparing the network approach to the other three approaches, the forest plot in Figure 3 indicates that the network approach performed better in terms of reducing zapping delay. The recently proposed network approaches include a time series model [19] that takes into account each of the stages where the service could be available, reducing the average delay error from the client to the headend. The results show that when the network is congested the model that best fits the data is the autoregressive Furthermore, to reduce zapping delay, [2] suggested using a hybrid delivery system that combines multicast and unicast-enabled services. The result presented shows that the hybrid approach outperforms the traditional method by reducing point-to-point queuing latency, end-to-end packet delay, and packet variance while maintaining a high throughput rate hence, reducing zapping delay tremendously.

\section{CONCLUSION}

It is naturally problematic for a network provider and or operators to avail reduced channel zapping delay as functionality to consumers in an IPTV system, but it is an inherent necessity on the quality of experience (QoE) of users. Channel switching delays can be traced to several sources and roll of techniques to combat this challenge. This study has therefore analyzed relevant and current techniques deployed for mitigating zapping delays. The meta-analyzed results showed that studies that captured 'network' delay components are capable of proper delay management and can reduce delay in channel zapping in an appropriate systematic manner than studies with 'all' delay components. Nevertheless, it has been proven experimentally in this study that virtual elimination of zapping delay may be made possible for a relevant chunk of channel switching requests. This further corroborates the fact that techniques involving channel switching request scheduler on the network may virtually eliminate latent zapping delays.

\section{REFERENCES}

[1] Quintero, M. A. R., Zachey, B., and Raake, A., "Annoyance and acceptability of video service responsiveness," Proceedings of Quality of Multimedia Experience (QoMEX), 2015 Seventh International Workshop, pp. 1-6, 2015, doi: 10.1109/QoMEX.2015.7148109.

[2] Adeliyi, T. T. and Olugbara, O. O., "Fast channel navigation of internet protocol television using adaptive hybrid delivery method," Journal of Computer Networks and Communications, 2018, doi: 10.1155/2018/2721950.

[3] Adeliyi, T. T. and Olugbara, O. O, "Reducing zapping delay in internet protocol television using a hybrid modular method," 2019 Conference on Information Communications Technology and Society (ICTAS), pp. 1-4, Mar. 2019, doi: 10.1109/ICTAS.2019.8703627.

[4] Begen, A. C., Perkins, C., and Ott, J., "On the use of RTP for monitoring and fault isolation in IPTV," IEEE Network, vol. 24, no. 2 pp. 14-19, 2010, doi: 10.1109/MNET.2010.5430139.

[5] Cha, M., Rodriguez, P., Moon, S. B., and Crowcroft, J., "On next-generation telco-managed P2P TV architectures," IPTPS, pp. 1-6, Feb. 2008.

[6] Friedrich, O., Thatmann, D., and Arbanowski, S., "An IPTV Service State API for converging managed and unmanaged IPTV infrastructures," Proceedings of Multimedia and Expo (ICME), 2010, pp. 1493-1498, doi: 10.1109/ICME.2010.5583284.

[7] Moustafa, H., and Zeadally, S., "Media networks: architectures, applications, and standards," CRC Press. pp. 1-555, 2012.

[8] Uilecan, I. V., Zhou, C., and Atkin, G. E., "Framework for delivering IPTV services over WiMAX wireless networks," 2007 IEEE International Conference on Electro Information Technology, pp. 470-475, 2007, doi: 10.1109/EIT.2007.4374532.

[9] Krogfoss, B., Sofman, L., and Agrawal, A, "Caching architectures and optimization strategies for IPTV networks," Bell Labs Technical Journal, vol. 13, no. 3, pp. 13-28, 2008, doi: 10.1002/bltj.20320.

[10] Škrbic, M., Šecic, N., and Varatanovic, M., “August. A unicast-based IPTV service control," 2010 Fifth International Conference on Systems and Networks Communications, IEEE, pp. 278-282, 2010, doi: 10.1109/ICSNC.2010.69.

[11] Alasaad, A., Gopalakrishnan, S., and Leung, V. C., "A hybrid approach for cost-effective media streaming based on prediction of demand in community networks," Telecommunication Systems, vol. 59, no. 3, pp. 329-343, 2015, doi: 10.1007/s11235-014-9939-7.

[12] Lee, E., Ku, J. Y., and Bahn, H., "An efficient hot channel identification scheme for IPTV channel navigation," IEEE Transactions on Consumer Electronics, vol. 60, no. 1, pp. 124-129, 2014, doi: 10.1109/TCE.2014.6780934.

[13] Merani, M. L., Natali, L., and Barcellona, C., "An IP-TV P2P streaming system that improves the viewing quality and confines the startup delay of regular audience," Peer-to-Peer Networking and Applications, vol. 9, no. 1, pp. 209-222, 2016, doi: 10.1007/s12083-014-0323-x. 
[14] Ghaderzadeh, A., Kargahi, M., and Reshadi, M., "ReDePoly: reducing delays in multi-channel P2P live streaming systems using distributed intelligence," Telecommunication Systems, pp. 1-16, 2017, doi: 10.1007/s11235-017-0336-x.

[15] Malkoş, S., Uccedil, E. and Akdeniz, R., "Improving QoE in multicast IPTV systems: Channel zapping times," Scientific Research and Essays, vol. 7, no. 35, pp. 3107-3113, 2012, doi: 10.5897/SRE12.182.

[16] Ghahfarokhi, B. S., Moghim, N. and Eftekhari, S., "Reducing channel zapping time in live TV broadcasting over content centric networks," Multimedia Tools and Applications, vol. 76, no. 22, pp. 23239-23271, 2017, doi: 10.1007/s11042-016-4037-3.

[17] Adeliyi, T. T. and Olugbara, O. O, "Designing two-list group program driven algorithm for channel navigation in internet protocol television," International Journal of Advanced Media and Communication, vol. 7, no. 4, pp. 251-263, doi: 10.1504/IJAMC.2019.105440.

[18] Chen, Y.-W. and Chiu, T.-T, "Minimizing Zapping Time in IPTV Based on User's Interest," International Journal of Computer and Communication Engineering, vol. 1, no. 2, p. 77, 2012, doi:10.7763/IJCCE.2012.V1.22.

[19] Ferro, R., Rodríguez, J. and Hernández, C., "Modelo en Series de Tiempo del Retardo del Cambio de Canal en una Transmission IPTV," Información tecnológica, vol. 26, no. 6, pp. 155-168, 2015, doi:10.4067/S071807642015000600017.

[20] Sarni, M., Hilt, B. and Lorenz, P., “A novel channel switching scenario in multicast IPTV networks," In: Proceedings of Networking and Services, 2009, ICNS'09, Fifth International Conference on, pp. 396-401, 2009, doi: 10.1109/ICNS.2009.52.

[21] Boyce, J. M. and Tourapis, A., Thomson Licensing S. A., "Method and apparatus enabling fast channel change for DSL system," U.S. Patent 9, 2015.

[22] Banodkar, D., Ramakrishnan, K., Kalyanaraman, S., Gerber, A. and Spatscheck, O, "Multicast instant channel change in IPTV systems," Proceedings of Communication Systems Software and Middleware and Workshops, 2008 , pp. 370-379, doi: 10.1109/COMSWA.2008.4554442.

[23] Mandal, S. K. and MBuru, M., "Intelligent pre-fetching to reduce channel switching delay in IPTV systems," Texas A \& $M$ University, 2008.

[24] Nikoukar, A., Hwang, I.-S., Liem, A. T. and Lee, J.-Y, "Mitigating the IPTV Zap time in enhanced EPON systems," Journal of Optical Communications and Networking, vol. 8, no. 6, pp. 451-461, 2016, doi: 10.1364/JOCN.8.000451.

[25] Cho, C., Han, I., Jun, Y. and Lee, H., "Improvement of channel zapping time in IPTV services using the adjacent groups join-leave method," Proceedings of Advanced Communication Technology, The 6th International Conference, 2004, doi: 10.1109/ICACT.2004.1293012.

[26] Lee, H., Kim, H. Y. and Lee, H.-K., "News package service based on TVAnytime metadata gathered from RSS," Proceedings of Consumer Electronics, IEEE International Symposium, pp. 1-6, 2007, doi: 10.1109/ISCE.2007.4382193.

[27] Kim, Y., Park, J. K., Choi, H. J., Lee, S., Park, H., Kim, J., Lee, Z. and Ko, K., "Reducing IPTV channel zapping time based on viewer's surfing behaviour and preference," Proceedings of Broadband Multimedia Systems and Broadcasting, pp. 1-6, 2008, doi: 10.1109/ISBMSB.2008.4536621.

[28] Ramos, F. M., Crowcroft, J., Gibbens, R. J., Rodriguez, P. and White, I. H., "Channel smurfing: minimising channel switching delay in IPTV distribution networks," Proceedings of Multimedia and Expo (ICME), 2010 IEEE International Conference, pp. 1327-1332, 2010, doi: 10.1109/ICME.2010.5583279.

[29] Ramos, F. M., Crowcroft, J., Gibbens, R. J., Rodriguez, P. and White, I. H., "Reducing channel change delay in IPTV by predictive pre-joining of TV channels," Signal Processing: Image Communication, vol. 26, no. 7, pp. 400412, 2011, doi: 10.1016/j.image.2011.03.005.

[30] Lee, Y., Lee, J., Kim, I. and Shin, H., "Reducing IPTV channel switching time using H, 264 scalable video coding," IEEE Transactions on Consumer Electronics, vol. 54, no. 2, pp. 912-919, 2008, doi: 10.1109/TCE.2008.4560178.

[31] Lee, C. Y., Hong, C. K. and Lee, K. Y.,” Reducing channel zapping time in IPTV based on user's channel selection behaviours," IEEE Transactions on Broadcasting, vol. 56, no. 3, pp. 321-330, 2010, doi: 10.1109/TBC.2010.2051494.

[32] Manzato, D.A. and da Fonseca, N. L, "A survey of channel switching schemes for IPTV," IEEE Communications Magazine, vol. 51, no. 8, pp. 120-127, 2013, doi: 10.1109/MCOM.2013.6576349.

[33] Khosroshahi, A. A., Yousefi, S. and Rahbar, A. G., "IPTV channel switching delay reduction through predicting subscribers' behaviors and preferences," Multimedia Tools and Applications, vol. 75, no. 11, pp. 6283-6302, 2016, doi: 10.1007/s11042-015-2572-y.

[34] Moher, D., Liberati, A., Tetzlaff, J., and Altman, D. G., "Preferred reporting items for systematic reviews and metaanalyses: the PRISMA statement," Int J Surg, vol. 8, no. 5, pp. 336-341, 2010, doi: 10.3736/jcim20090918.

[35] Tam, W. W., Tang, A., Woo, B. and Goh, S. Y., "Perception of the preferred reporting items for systematic reviews and meta-analyses (PRISMA) statement of authors publishing reviews in nursing journals: a cross-sectional online survey," BMJ open, vol. 9, no. 4, p. e026271, 2019, doi: 10.1136/bmjopen-2018-026271.

[36] Zhang, P., and Liu, B., "Differentiation among glioblastomas, primary cerebral lymphomas, and solitary brain metastases using diffusion-weighted imaging and diffusion tensor imaging: A PRISMA-compliant meta-analysis, "ACS Chemical Neuroscience, vol. 11, no. 3, pp. 477-483, 2020, doi: 10.1021/acschemneuro.9b00698.

[37] Munn, Z., Moola, S., Riitano, D., and Lisy, K., "The development of a critical appraisal tool for use in systematic reviews addressing questions of prevalence," International journal of health policy and management, vol. 3, no. 3, pp. 123, 2014, doi: 10.15171/ijhpm.2014.71. 
[38] Fabrizi, F., Cerutti, R., Donato, F. M. and Messa, P., "HBV infection is a risk factor for chronic kidney disease: Systematic review and meta-analysis," Revista Clinica Espanola, 2020, doi: 10.1016/j.rce.2019.10.010.

[39] Fahmy, T. and Bellétoile, A., "Algorithm 983: Fast Computation of the Non-Asymptotic Cochran's Q Statistic for Heterogeneity Detection," ACM Transactions on Mathematical Software (TOMS), vol. 44, no. 2, pp. 1-12, 2017, doi: 10.1145/3095076.

[40] Lin, L. and Chu, H., "Quantifying publication bias in meta-analysis," Biometrics, vol. 74, no. 3, pp. 785-794, 2018, doi: 10.1111/biom.12817.

[41] Lin, L., Chu, H., Murad, M. H., Hong, C., Qu, Z., Cole, S. R. and Chen, Y., "Empirical comparison of publication bias tests in meta-analysis," Journal of general internal medicine, vol. 33, no. 8, pp. 1260-1267, 2018, doi: 10.1007/s11606-018-4425-7.

[42] Mathur, M. B. and VanderWeele, T. J, "Estimating publication bias in meta-analyses of peer-reviewed studies: A metameta-analysis across disciplines and journal tiers," Research Synthesis Methods, 2020, doi.org/10.1002/jrsm.1464.

\section{BIOGRAPHIES OF AUTHORS}
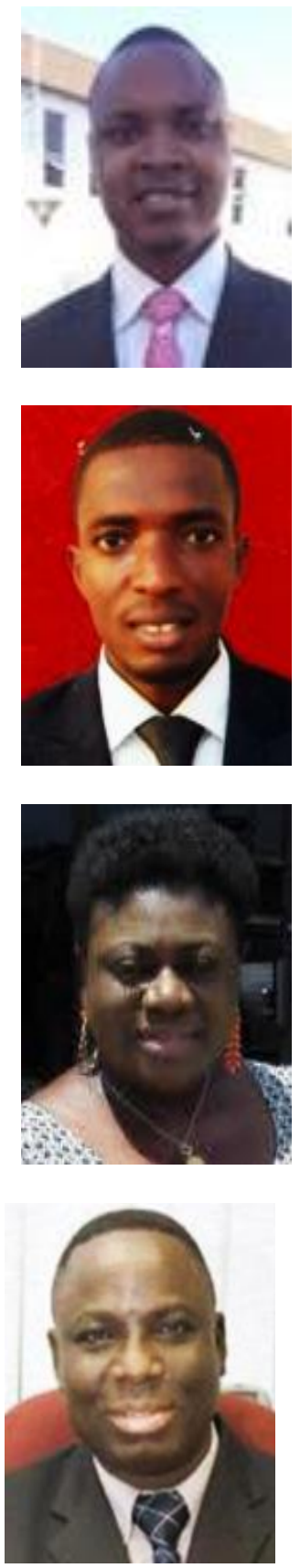

Timothy T. Adeliyi, is a Lecturer in the Department of Information Technology at the Durban University of Technology. He received his $\mathrm{PhD}$ in Information Technology, MSc in Data Networks \& Security and BSc in Information Technology. His research inclinations are multimedia systems, machine learning, computer networks and security. He is a member of the ICT and Society Research Group of Durban University of Technology.

Ropo E. Ogunsakin, is a statistician and postdoctoral fellow at Durban University of Technology, South Africa. His research interest is mostly based on statistical modelling and meta-analysis. He is a member of the ICT and Society Research Group of Durban University of Technology.

Marion O. Adebiyi, is a faculty of the Department of Computer Science at Landmark University, Omu-Aran, Nigeria. She holds a B.Sc Degree from University of Ilorin, Ilorin Nigeria. She had her M.Sc. and PhD Degree in Computer Science from Covenant University, Nigeria respectively. Her research interests include Bioinformatics of Infectious (African) Diseases/ Population, Organism's Inter-pathway analysis, High throughput data analytics, Homology modelling and Artificial Intelligence. She has published widely in local and international reputable journals. She is a member of the Nigerian Computer Society (NCS), the Computer Registration Council of Nigeria (CPN) and IEEE member.

Oludayo O. Olugbara, holds a PhD in Computer Science, University of Zululand, 2008, M.Sc. in Mathematics (Computer Science Specialization), University of Ilorin, Nigeria, 1995, and a B.Sc. (Honors) in Mathematics (First Class Honors), University of Ilorin, Nigeria, 1991. His research inclinations are Machine Learning, Computer Games, Image Processing, Grid/Cloud computing and Scientific Computing. 